\title{
An Optical Receiver Post Processing System for the Integrated Radio and Optical Communications Software Defined Radio Test Bed
}

\author{
Jennifer M. Nappier ${ }^{1}$, Roger P. Tokars ${ }^{2}$ and Adam C. Wroblewski ${ }^{3}$ \\ NASA Glenn Research Center, Cleveland, Ohio 44135
}

\begin{abstract}
The Integrated Radio and Optical Communications (iROC) project at the National Aeronautics and Space Administration's (NASA) Glenn Research Center is investigating the feasibility of a hybrid radio frequency (RF) and optical communication system for future deep space missions. As a part of this investigation, a test bed for a radio frequency (RF) and optical software defined radio (SDR) has been built. Receivers and modems for the NASA deep space optical waveform are not commercially available so a custom ground optical receiver system has been built. This paper documents the ground optical receiver, which is used in order to test the RF and optical SDR in a free space optical communications link.
\end{abstract}

\section{Nomenclature}

$K_{s} \quad=$ signal photon counts per signal slot

$K_{b} \quad=$ background photons per slot

\section{Introduction}

$\mathrm{T}$

HE Integrated Radio and Optical Communications (iROC) ${ }^{1}$ project at the National Aeronautics and Space Administration's (NASA) Glenn Research Center is developing technologies which will support a hybrid radio frequency (RF) and optical communication system targeted for deep space missions. As a part of this project, an integrated RF and optical software defined radio $^{2}$ (SDR) has been built using commercial-off-the-shelf (COTS) components. The SDR performs offset quadrature phase shift keying (OQPSK) for the RF modulation and pulse position modulation (PPM) for the optical modulation. Commercial modems are commonly used in testing RF SDRs, but no commercial receiver exists to test optical PPM in the single photon counting regime. Custom PPM receivers have been used for the Lunar Laser Communications Demonstration Project ${ }^{3}$ (LLCD), including a post processing software receiver ${ }^{4}$ built by the Jet Propulsion Laboratory (JPL). The optical receiver described in this paper uses commercial-off-the-shelf hardware in combination with post processing software to receive, demodulate, and decode the received PPM signal.

The receiver architecture is described in detail in this paper. Section II describes the requirements for the optical receiver. The receiver architecture is described in Section III. Results are presented in Section IV and the conclusion and future work is discussed in Section V.

\section{Optical Receiver Requirements}

The requirements for the optical receiver are as follows:

1. Receive, demodulate, and decode a free space optical link operating at a data rate of $2 \mathrm{Mbps}$ with a 16-ary serially concatenated pulse position modulated ${ }^{5}$ (SCPPM) rate $1 / 2$ code, a 16 symbol frame acquisition sequence, and 4 inter symbol guard time slots.

\footnotetext{
${ }^{1}$ Aerospace Technologist in Data Systems, Information and Signal Processing Branch, 21000 Brookpark Road MS 54-1.

${ }^{2}$ Research Aerospace Technologist in Electro-Optical Sensor Systems, Optics and Photonics Branch, 21000 Brookpark Road MS 77-1.

${ }^{3}$ Research Aerospace Technologist in Measurement and Instrument Systems, Optics and Photonics Branch, 21000 Brookpark Road MS 77-1.
}

American Institute of Aeronautics and Astronautics 
2. Continuously monitor the received signal power and the photomultiplier tube (PMT) current while collecting received data.

\section{Receiver Architecture}

The receiver hardware can be broken down into three main subsystems - the optics, the analog, and the digital subsystem. A block diagram of the receiver is shown in Figure 1. The subsystems are described in the following sections.

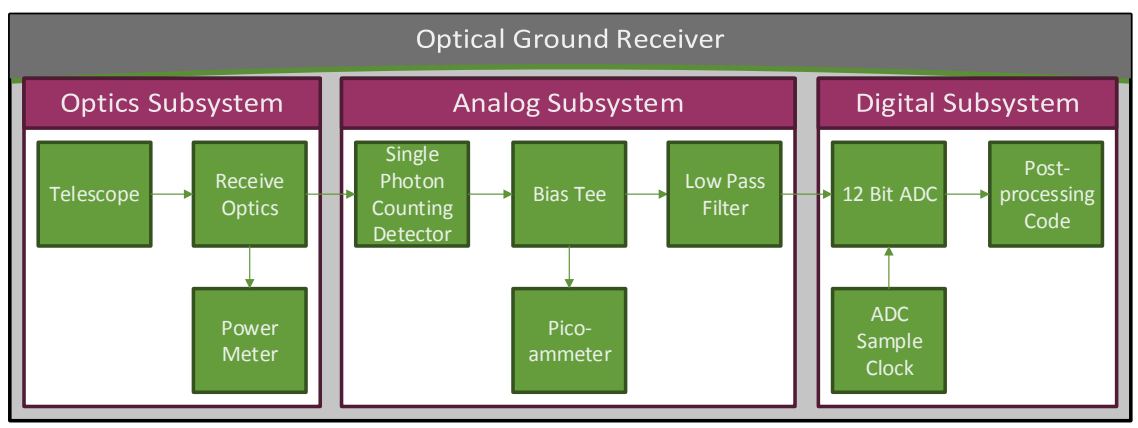

Figure 1. Optical Receiver Block Diagram.

\section{A. Optics Subsystem}

The optical receiver subsystem, illustrated in Figure 2, has been designed to collect and deliver a $\lambda=1550$ $\mathrm{nm}$ modulated laser transmission to a photon detector. The 5 inch diameter receive aperture is based on a COTS Cassegrain telescope which focuses the transmitted modulated laser light through series of optics before reaching the photon detector. After being focused through the Cassegrain telescope, the light passes through a $1550 \mathrm{~nm}$ band pass filter to reduce the magnitude of other irrelevant wavelengths. A collimating lens reshapes the collected light into a beam which is split by a 90:10 beam splitter. The higher power (90\%) light beam is directed into a fiber coupled power meter which allows for monitoring of the system's alignment as well as approximate power into the photon detector. The lower power (10\%) light beam is focused into a $200 \mu \mathrm{m}$ OD fiber which delivers the modulated laser light into the photon detector.

Coarse alignment of the system is performed using visible light $(\lambda=635 \mathrm{~nm})$. During coarse alignment, the photon detector is blocked, the band pass filters are removed, and full access to all optics exists. Once complete, the optics are reinstalled and special care is taken to house the optical path in a light-tight enclosure to minimize data corruption due to stray light. Prior to operation of the photon detector, a safe magnitude of laser light power is established based on measurements by the power meter.

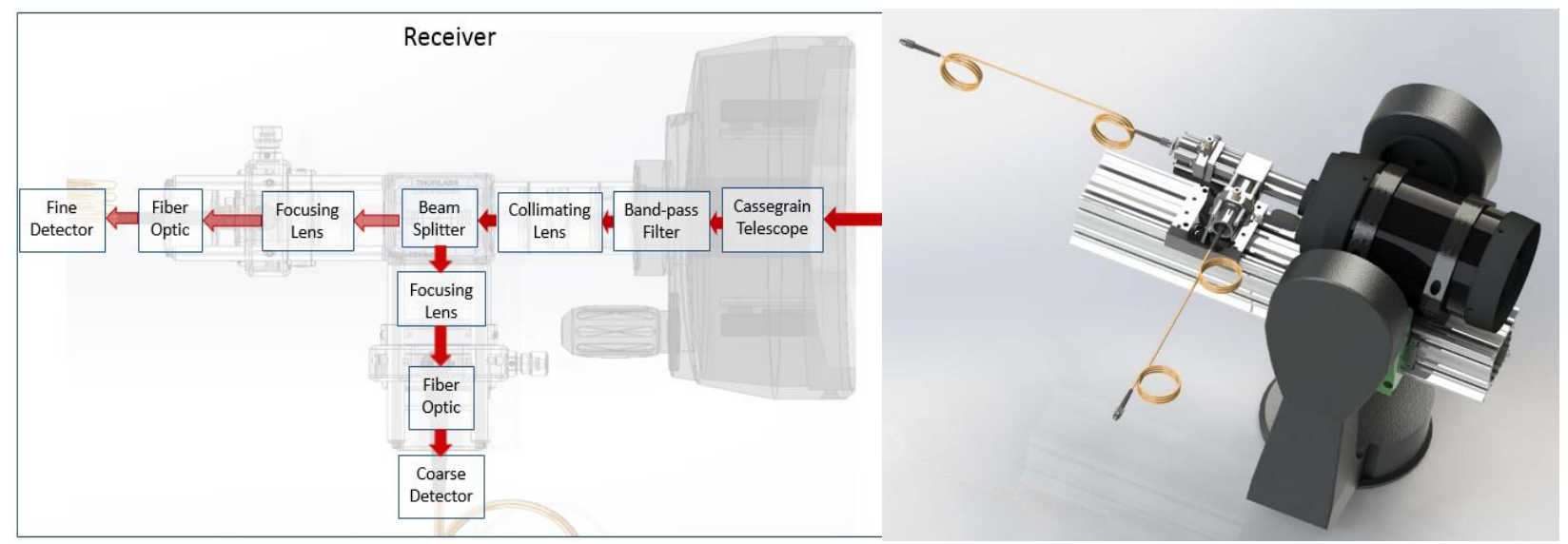

Figure 2. iROC receiver block diagram describing optical train. 


\section{B. Analog Subsystem}

The purpose of the analog subsystem is to convert the incoming light into electrical pulses compatible with the digital receiver. An electrical pulse is output from the PMT when a photon is incident on the detector surface. The low pass filter broadens the electrical pulse to enable slower analog to digital converter (ADC) sample rates. A bias tee allows a picoammeter to simultaneously monitor the PMT current while passing the pulses through to the digital receiver. The Pentek ADC samples the PMT pulses which will be demodulated and decoded using the post processing software.

\section{Photo Multiplier Tube Characterization}

A Hamamatsu PMT was chosen as the single photon counting detector. This PMT allows the receiver to be architected to operate in the single photon counting regime, but is available commercially for a significantly lower cost than single photon detectors which must be cryocooled to $4 \mathrm{~K}$. The operating point of a PMT is set by changing the supply voltage. In order to determine the ideal PMT supply voltage for this receiver system, several characterization tests were completed. The tests included dark current measurements, pulse height distribution histograms, and characterization of the signal to noise ratio. Each test was completed across the supply voltage range of the PMT.

The dark current of the PMT was measured and compared to the manufacturer data. Results can be seen in Figure 3 and were consistent with the manufacturer data. As the PMT supply voltage increases, so does the dark current. It is important to note that when the PMT is powered on, the photocathode is gradually cooled to a low temperature set point. The dark current continues to decrease until the temperature stabilizes. Dark current measurements should be made after the PMT has been powered on for at least one hour.

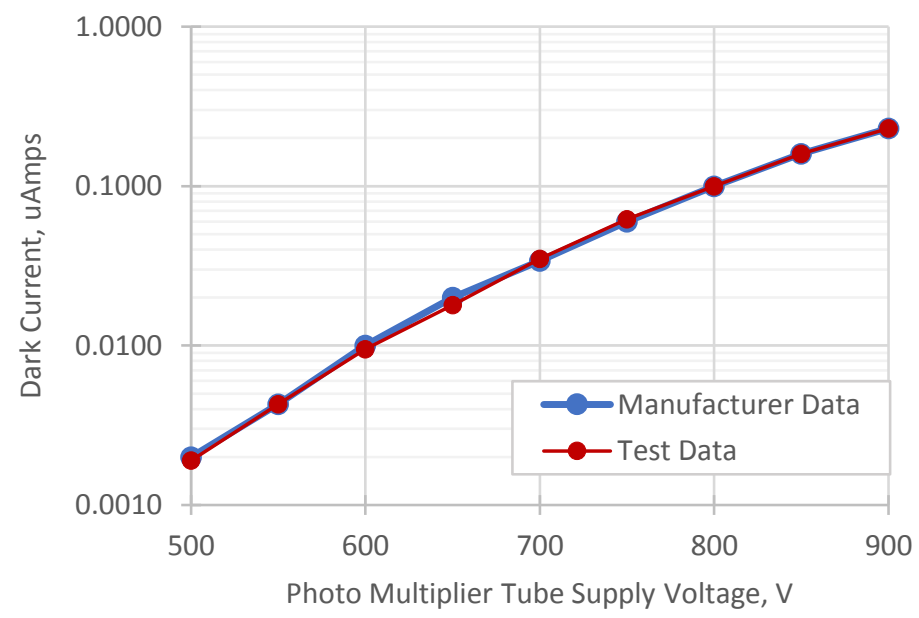

Figure 3. PMT Dark Current Characterization.

The pulse height distribution was measured for PMT supply voltages between $550 \mathrm{~V}$ to $700 \mathrm{~V}$ without light input (dark counts) and with a constant light level of $10 \mathrm{pW}$. The measurement was done with the low pass filter in the system. Results at supply voltages of $575 \mathrm{~V}$ and $675 \mathrm{~V}$ are shown in Figure 4 and Figure 5. In each figure, the lower red histogram is from only dark current while the higher blue histogram is from the $1550 \mathrm{~nm}$ light and dark current. It can be seen that at both $575 \mathrm{~V}$ and $675 \mathrm{~V}$, the dark current histogram and the histogram from $1550 \mathrm{~nm}$ light follow the same basic trend. At $575 \mathrm{~V}$, the pulse heights ranged from $2-8 \mathrm{mV}$ while at $675 \mathrm{~V}$, the range was $2-25 \mathrm{mV}$. The pulse height distribution is important to characterize as it allows the threshold level for photon detections in the ADC card and post processing code to be set properly.

American Institute of Aeronautics and Astronautics 


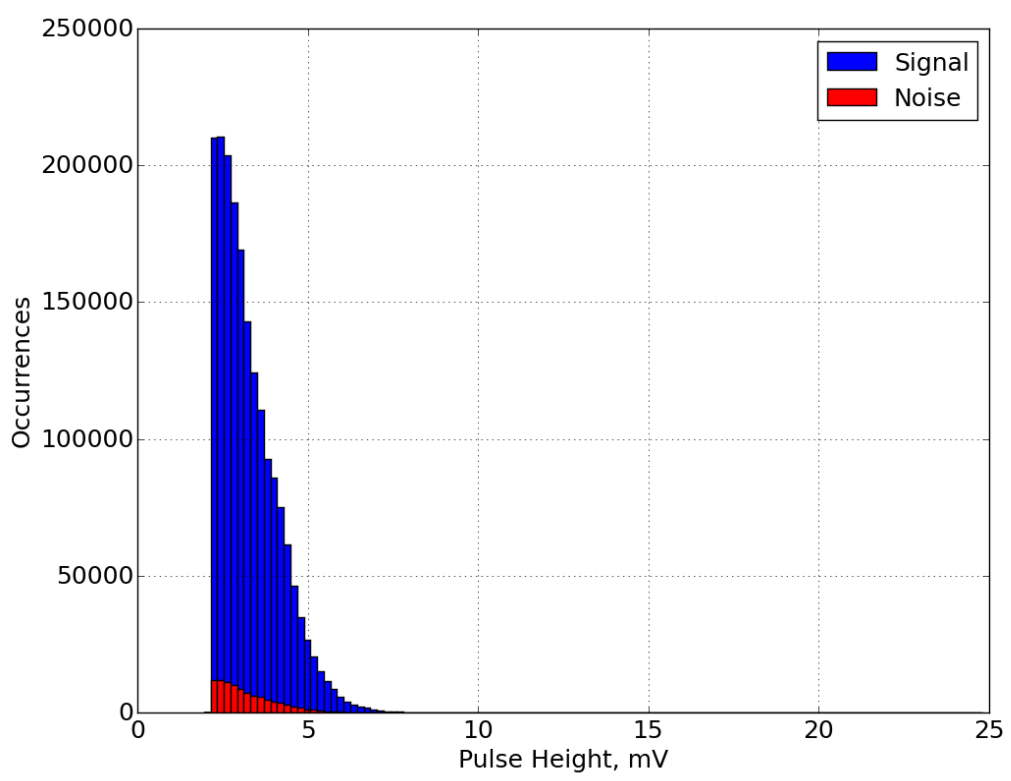

Figure 4. PMT Pulse height distribution, supply = 575 V.

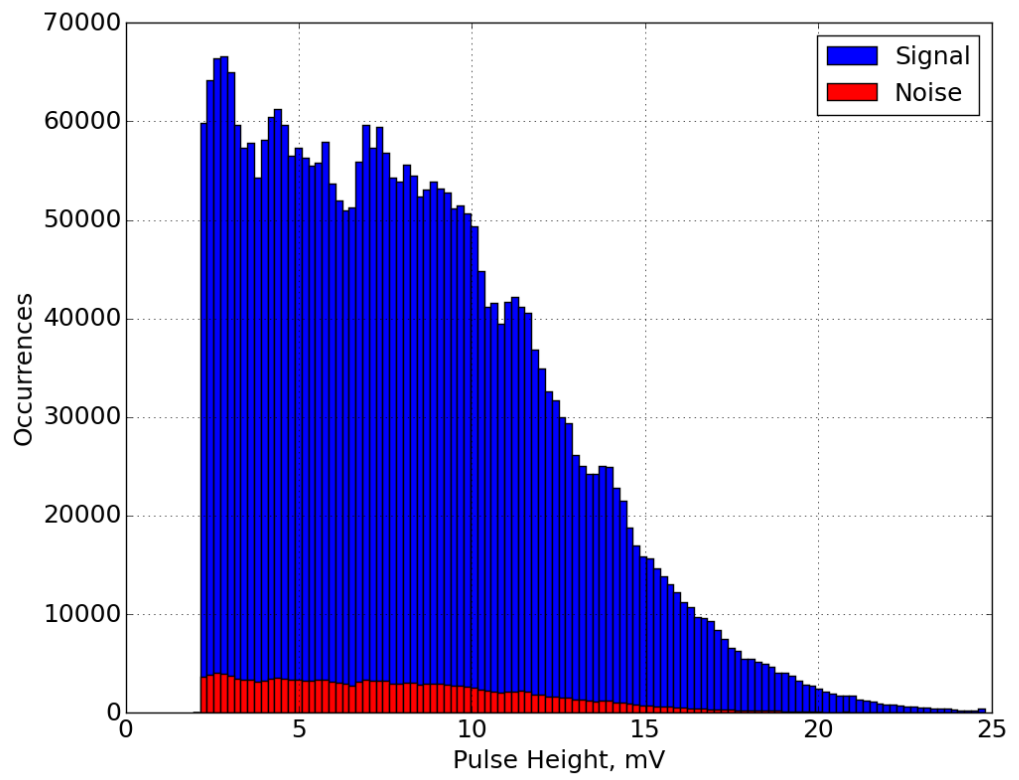

Figure 5. PMT pulse height distribution, supply $=675 \mathrm{~V}$.

Finally, varying levels of light were input into the PMT and counts were measured in order to calculate the signal to noise $(\mathrm{S} / \mathrm{N})$ ratio at each supply voltage. Figure 6 shows the $\mathrm{S} / \mathrm{N}$ ratio for input light levels ranging from 10 $\mathrm{pW}$ to $71 \mathrm{pW}$. It can be seen that at a PMT supply voltage of $550 \mathrm{~V}$, the $\mathrm{S} / \mathrm{N}$ ratio is maximized. This is expected, because at the lower supply voltage, the PMT can accept higher levels of light without exceeding the maximum current draw of $1 \mu \mathrm{A}$. The operating point for the PMT was determined to be at a supply voltage $575 \mathrm{~V}$ because it has an excellent $\mathrm{S} / \mathrm{N}$, but also has pulse heights which are above the noise floor of the Pentek A/D card.

American Institute of Aeronautics and Astronautics 


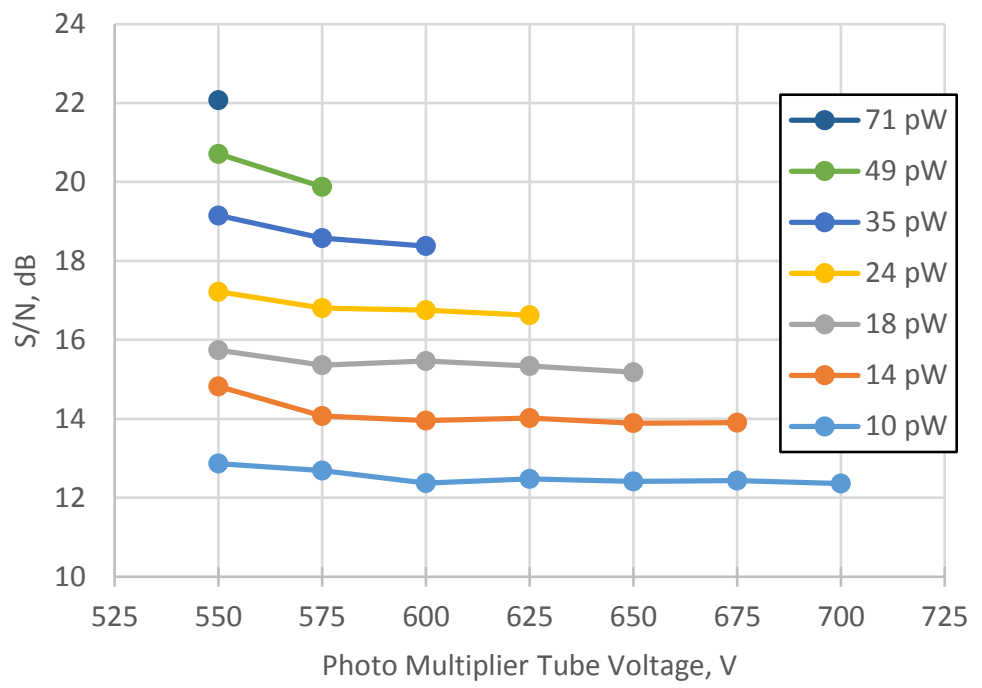

Figure 6. PMT S/N ratio.

\section{Digital Subsystem}

The digital subsystem consists of a custom built computer running Linux for the operating system. The computer houses a Pentek high speed 12 bit ADC card which is capable of continuously streaming data to the hard drive over the PCIe bus at rates up to $600 \mathrm{Msamples} / \mathrm{s}$. The Pentek card is driven by an external clock which is generated by a signal generator. The $600 \mathrm{Msamples} / \mathrm{s}$ rate sufficient to sample the pulses coming out of the PMT at greater than 2 samples per pulse. In order to achieve a sample rate of $600 \mathrm{Msamples} / \mathrm{s}$, the 8 least significant bits from the Pentek card were saved with timestamps when they exceeded the pulse threshold. This algorithm decreases the number of writes to the solid state hard drive when saving samples, which is the slowest part of the system. The PMT samples are saved to the computer hard drive for post processing. The computer is capable of saving enough samples to measure a code word error rate of $10^{-3}$ with 100 received errors. This is approximately $228 \mathrm{~GB}$ or 380 seconds of data.

The post processing receiver code $^{4}$ was originally developed for the Lunar Laser Communications Demonstration (LLCD) by JPL for use at the Lunar Laser Optical Communications Telescope Laboratory (OCTL) Terminal (LLOT). The post processing code was modified to perform slot synchronization using the inter symbol guard time and code word alignment using the frame acquisition sequence. This modification is necessary for the high photon efficiency SCPPM waveform to be used in deep space.

\section{Results}

This optical receiver system has been used to demodulate and decode the $2 \mathrm{Mbps}$, SCPPM-16, $1 / 2$ rate code, signal transmitted over a free space optical link. The optical signal was generated using the iROC RF and optical SDR in $^{2}$ combination with both a high extinction ratio (specified at $>40 \mathrm{~dB}$ ) and lower extinction ratio (specified at $20 \mathrm{~dB}$ ) electro-optic modulator. The link was setup indoors in a clean room high bay. The PMT was run with a 575 supply voltage and a maximum current draw of close to $0.9 \mu \mathrm{A}$. The Pentek ADC was used to capture data files ranging in size from $1 \mathrm{~GB}$ to $250 \mathrm{~GB}$ to be post processed. The data was decoded and $\mathrm{K}_{\mathrm{s}}, \mathrm{K}_{\mathrm{b}}$, and the code word error rate were measured. $\mathrm{K}_{\mathrm{s}}$ ranged from 3.1-4.3 photons/signal slot and $\mathrm{K}_{\mathrm{b}}$ ranged from 0.037-0.0025 photons/slot. This variation was accomplished by adjusting the bias voltage of the electro-optic modulator to change the extinction ratio. When $\mathrm{K}_{\mathrm{b}}$ was 0.0025 , the electro optic modulator was biased for the highest extinction ratio and the noise in the system was due to the dark current of the PMT. The data is presented in Table 1. A graph of $\mathrm{K}_{\mathrm{s}}$ and $\mathrm{K}_{\mathrm{b}}$ with exponential averaging over 60 seconds can be seen in Figure 7. The figure shows that they both fluctuate a little bit but remain relatively constant over time.

American Institute of Aeronautics and Astronautics 
The current from the picoammeter was found to be a more consistent measurement of the light received than the coupled port from the optics due to changing fiber coupling into the $90 \%$ and $10 \%$ splits. Therefore, the light received at the PMT was calculated from the current, PMT supply voltage, and gain.

Figure 8 shows a graph of the $\mathrm{K}_{\mathrm{s}}$ with exponential averaging and the PMT current over a time period of 60 seconds. It can be seen that the PMT current and $\mathrm{K}_{\mathrm{s}}$ trend similarly.

Table 1.Optical Link Testing Results.

\begin{tabular}{|l|l|l|l|l|l|l|c|c|}
\hline $\begin{array}{c}\text { Data } \\
\text { Samples } \\
(\mathbf{G B})\end{array}$ & $\begin{array}{c}\text { Coded } \\
\text { Words } \\
\text { Processed }\end{array}$ & $\begin{array}{c}\text { Code } \\
\text { Word } \\
\text { Errors }\end{array}$ & $\begin{array}{c}\text { Code } \\
\text { Word } \\
\text { Error } \\
\text { Rate }\end{array}$ & $\begin{array}{c}\mathbf{K}_{\mathbf{s}} \\
\text { (photons/signal } \\
\text { slot) }\end{array}$ & $\begin{array}{c}\mathbf{K}_{\mathbf{b}} \\
\text { (photons/slot) }\end{array}$ & $\begin{array}{c}\text { Average } \\
\text { PMT } \\
\text { Current } \\
(\boldsymbol{\mu} \mathbf{A})\end{array}$ & $\begin{array}{c}\text { PMT } \\
\text { Supply } \\
\text { Voltage } \\
\text { (Volts) }\end{array}$ & $\begin{array}{c}\text { Specified } \\
\text { Electro } \\
\text { Optic } \\
\text { Modulator } \\
\text { Extinction } \\
\text { Ratio }\end{array}$ \\
\hline 100 & 43,900 & 14 & 0.00032 & 3.6 & 0.037 & 0.91 & 575 & $20 \mathrm{~dB}$ \\
\hline 100 & 43,900 & 4 & 0.000091 & 4.3 & 0.0025 & 0.93 & 575 & $>40 \mathrm{~dB}$ \\
\hline 100 & 43,900 & 292 & 0.0067 & 3.3 & 0.016 & $\sim 0.9^{*}$ & 575 & $>40 \mathrm{~dB}$ \\
\hline 250 & 109,756 & 270 & 0.0025 & 3.4 & 0.014 & $\sim 0.9^{*}$ & 575 & $>40 \mathrm{~dB}$ \\
\hline 1 & 428 & 2 & 0.0047 & 3.1 & 0.016 & $\sim 0.9^{*}$ & 575 & $>40 \mathrm{~dB}$ \\
\hline
\end{tabular}

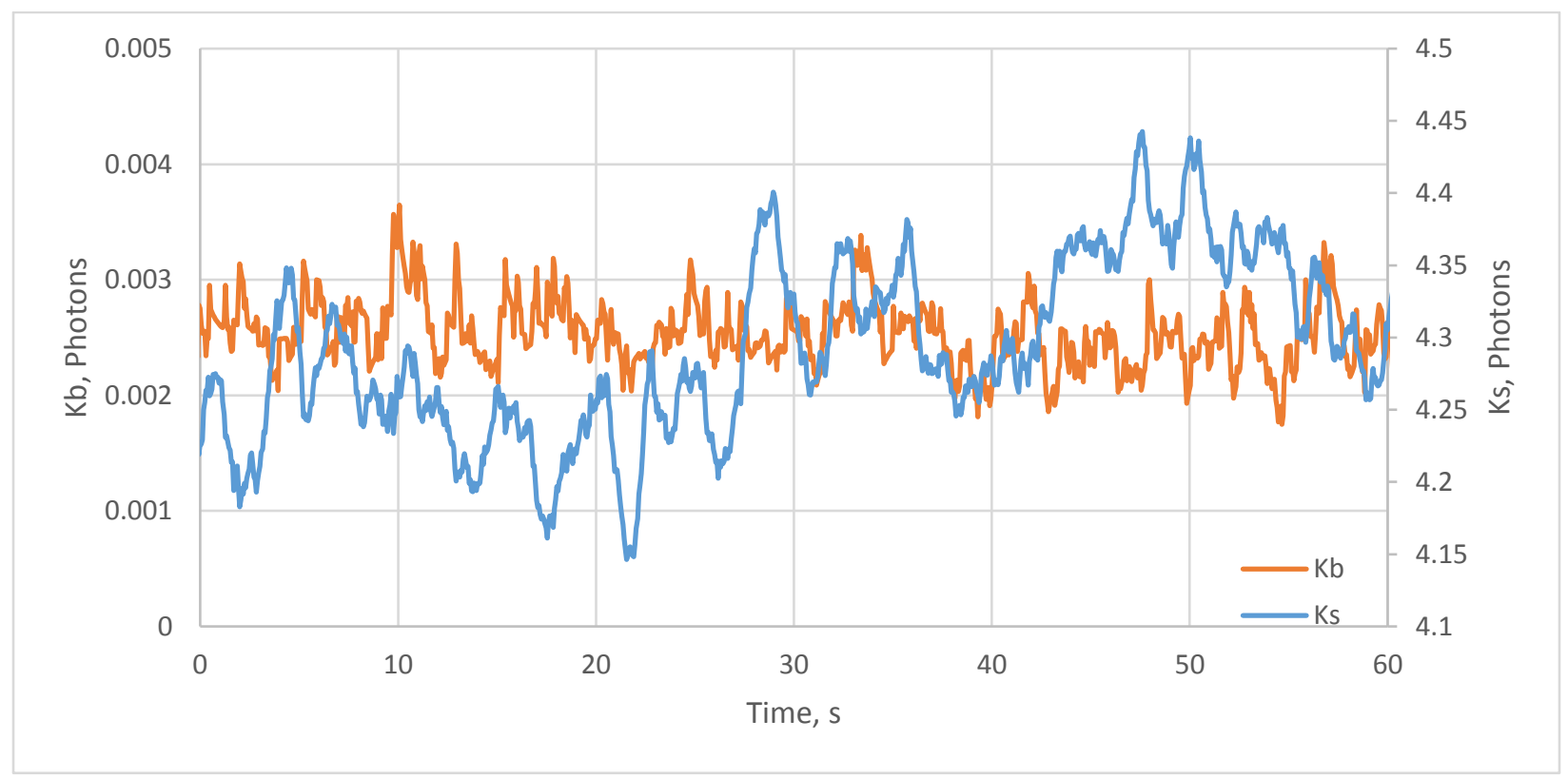

Figure 7. Ks and Kb. ${ }^{*}$ The PMT current was set to $0.90-0.95 \mathrm{uA}$ as part of the testing procedure, but data was not recorded for the duration
of the test. 


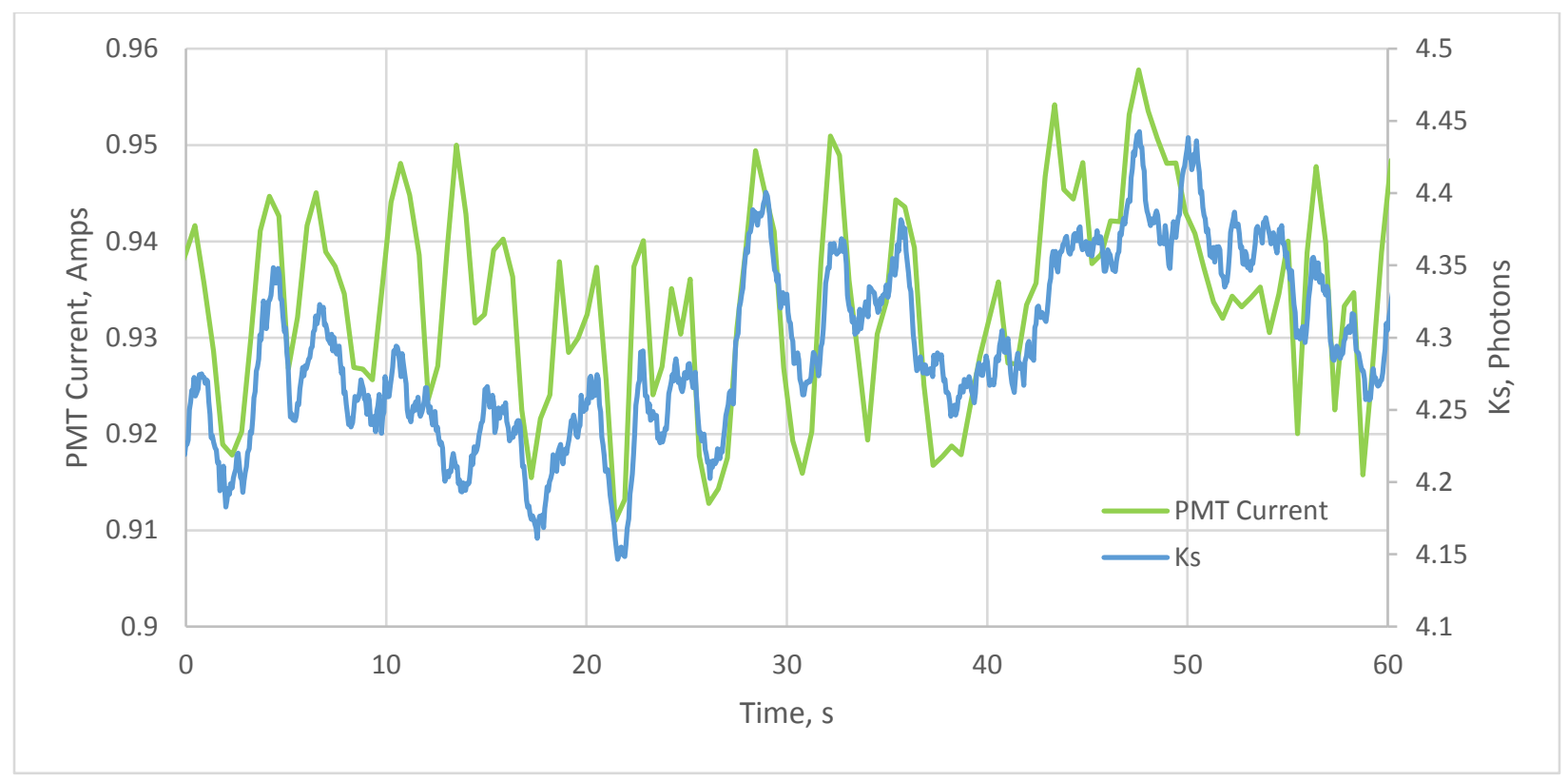

Figure 8. PMT Current and Ks.

\section{Conclusion}

A post processing receiver system was developed from COTS components for the iROC project at NASA Glenn Research center. The receiver system consisted of the optics, analog, and digital subsystems. Initial results show that the received PMT current and Ks trend in similar directions. The receiver system has been used to post process file sizes that are up to $250 \mathrm{~GB}$. The receiver system will be used in the future to conduct free space optical link tests under varying signal and noise conditions.

\section{Acknowledgements}

The authors would like to thank the NASA iROC team members for their work on the optical receiver post processing system, including Mr. Daniel Zeleznikar, Mr. Brian Tomko, and Ms. Caitlyn Harrington. This work was performed under the NASA SCaN Program at the NASA Glenn Research Center.

\section{References}

${ }^{1}$ D. Raible, R. Romanofsky, J. Budinger, J. Nappier, A. Hylton, A. Swank, and A. Nerone. "On the Physical Realizability of Hybrid RF and Optical Communications Platforms for Deep Space Applications." AIAA International Communications Satellite Systems Conference, AIAA, San Diego, CA, 2014.

${ }^{2}$ J. Nappier, D. Zeleznikar, A. Wroblewski, R. Tokars, B. Schoenholz, and N. Lantz. "A COTS RF/Optical Software Defined Radio for the Integrated Radio and Optical Communications Test Bed." AIAA International Communications Satellite Systems Conference, AIAA, Cleveland, OH, 2016.

${ }^{3}$ D. Boroson, B. Robinson, D. Murphy, D. Burianek, F. Khatri, J. Kovalik, Z. Sodnik, D. Cornwell. "Overview and Results of the Lunar Laser Communication Demonstration." Proceedings of the Free-Space Laser Communication and Atmospheric Propagation XXVI Conference, Vol. 8971, SPIE, 2014.

${ }^{4}$ M. Srinivasan, K. Birnbaum, M. Cheng, and K. Quirk. "A Post-Processing Receiver for the lunar Laser Communications Demonstration Project.” SPIE Photonics West, San Francisco, CA, February 2013.

${ }^{5}$ B. Moison and J. Hamkins. "Coded Modulation for the Deep-Space Optical Channel: Serially Concatenated Pulse Position Modulation.” The Interplanetary Network Progress Report, vol. 42-161, May 2005.

American Institute of Aeronautics and Astronautics 